\title{
Should HPV Vaccine be Mandated as Part of School Entry Requirements?
}

\author{
Fadi Al Daoud* \\ Trauma research coordinator, Hurley Medical Center, USA
}

Received: June 22, 2018; Published: July 02, 2018

*Corresponding author: Fadi Al Daoud, Trauma research coordinator, Hurley Medical Center,Michigan, USA

Abbreviations: HPV: Human Papilloma Virus; ACIP: Advisory Committee on Immunization Practices; Pap: Papanicolaou; FVC: Federal Vaccines for Children

\section{Introduction}

Human Papillomavirus (HPV) is the major cause of cervical cancer, in addition, this virus can cause different kinds of cancers. It is also responsible for genital warts [1]. The national Advisory Committee on Immunization Practices (ACIP) has some recommendations since June 2006, that routine HPV vaccination should be given to girls between ages 11 and 12, and it is now recommended for males as well. Debate about whether or not to mandate girls and boys to be vaccinated against HPV is continuing [2]. Data from the Centers for Disease Control and Prevention [3] shows that HPV infects almost 6.2 million people each year in the United States [1]. The majority of HPV strains do not have any symptoms and resolve spontaneously. There is no curative treatment for HPV virus. However, there is treatment for health problems related to this virus.There are more than 30 species and strains of HPV that are responsible for infecting more than $50 \%$ of sexually active people during their lifetime [1]. Here is data from CDC showing the approximate number of different cancers associated with HPV in USA per year.

10,800 cervical cancers, 7,400 head and neck (oral cavity and oropharynx) cancers (5,700 in men and 1,700 in women)3,000 anal cancers (1,900 in women and 1,100 in men), and 2,300 new cases of vulvar cancer. Penile and vaginal cancers were relatively rare, striking about 800 men and around 600 women per year [3]. Cervical cancer is considered the second killing cancer among women worldwide. In the United States, it is responsible for the death of almost 3,700 women out of nearly 10,000 women who have cervical cancer each year [1]. However, this number is considered less than other countries that have a high percentage of cervical cancer. This is because of the regular Papanicolaou (Pap) screening test that can detect this cancer. A report from the American Cancer Society, shows that cervical cancer is usually treatable with early detection [1]. The FDA has approved two vaccines for HPV in early 2012. GlaxoSmithKline's Cervix, against HPV strains 16 and 18 and Merck's Gardasil, which prevents from HPV strains 6, 11, 16 and 18. Almost $90 \%$ of genital warts and $70 \%$ of cervical cancer cases are caused by these four strains of HPV [1].

HPV vaccines can be given starting at 9 years of age, followed by two booster doses. These three doses of HPV vaccine proved to be very effective against the different strains of HPV [4]. Currently jurisdictions in three states, Rhode Island, Virginia, and District of Columbia require HPV vaccines for school attendance. The Department of Health in Rhode Island announced in July 2015 that the HPV vaccine is one of the mandated vaccines for seventh grade students starting in September of 2015. The Department of Health in this state was able to do this without legislative action. Virginia legislature also mandated HPV vaccine as one of school vaccine requirements in 2007. On the other hand, D.C. in addition to 24 states introduced legislation bills in 2007 to mandate the HPV vaccine for school (California and Maryland withdrew their bills). DC's bill was enacted, and HPV vaccine started to be one of the requirements for school entrance during this year [1]. Many parents have stigma towards giving HPV vaccination to their children as they relate HPV to sex.

They believe that it is not necessary to give this vaccine to their children as they are too young to start sex even when they are in high school. In fact, data from repeated surveys shows that $50 \%$ of high school students have had sex [4]. Parents also are worried about the safety of HPV vaccine though this vaccine have been used for many years without proven side effects. However, once parents are not convinced about this vaccine, and since it is not mandatory for school, they just skip it entirely [4]. There are some critiques of HPV vaccination use. By giving girls and boys this 
vaccine, they may have a false belief that they are protected from sexually transmitted diseases and will lead to sexual disinhibition. People who criticize this vaccine, think that children are already taking too many vaccinations, and no need for extra shots, they also have doubt about the long-term effectiveness of this vaccine that extra doses need to be given in the future [5]. There are also some arguments against mandating HPV vaccine as part of schoolentry requirements. Some people think this process is an intrusion on parental autonomy; and implementing a vaccine that prevents against a non-casually transmitted disease should not be mandated.

Others think that the vaccine is expensive where people sometimes can't afford paying for it, and this should not be mandated until it is fully covered by insurance or Medicaid [5]. Given the serious nature of cervical cancer and given the information that it's occurrence is mainly linked to HPV infection, in addition to the different cancers that are related to this virus, it is imperative to give this vaccine to all school children unless they have some medical issues that prevent them from taking it to protect them against this virus and to reduce the risk of developing cancers related to it in future. Though there are some criticisms and arguments against HPV vaccine itself and against mandating it as part of school entry requirements, providing good education to the parents about this virus and about the long-termdevastating outcomes of being infected with HPV, this will reduce the stigma towards giving HPV vaccine to their children. For those who are skeptical about the cost of this vaccine, the CDC announced the availability of HPV vaccine in all 50 states through the Federal Vaccines for Children (VFC) program [1].

For people who are also skeptical about the effectiveness of HPV vaccines, a study shows that the maximum prevalence of HPV infection was reduced from $39 \%$ to $33 \%$ with $15 \%$ vaccination coverage, whereas the peak prevalence reduced to $7 \%$ with $90 \%$ coverage. The cumulative incidence of cervical cancer over 25 -year period is 27 cases per 1000 persons, with 90\% of HPV vaccine coverage, the cumulative incidence is 3 cases per 1000 persons. This reflects a relative reduction of $89 \%$ of the incidence of cervical cancer [6]. Vaccines including HPV are medical treatment. At the first glance, it looks sometimes odd to force parents to give their children a medical treatment. However, vaccine is different from most of medical treatments because it is a biological treatment that reduces the risk of transmitting vaccine-preventable diseases to other people [4]. It is very important to understand that when requiring vaccines including HPV for school entry, children are not only provided an education, they are also provided the best chance for a healthy future [5].

\section{References}

1. (2015) National Institute of Health NIH, HPV and Cancer.

2. (2017) National conference of state legislature NCSL, HPV Vaccine State Legislation and Statutes.

3. (2017) Center for Disease Control and Prevention CDC,Human Papillomavirus (HPV) and Cancer.

4. MC Carthy (2015) Why Public Schools Should Require the HPV Vaccine C, Harvard Health Publishing.

5. Haber G, Malow RM, ZimetGZ (2007) The HPV Vaccine Mandate Controversy. Journal of Pediatric and adolescent gynecology 20(6): 325333.

6. TracyL, Gaff HD, Burgess C, Sow S, Gravatt PE, et al. (2011) Estimating the Impact of Human Papillomavirus (HPV) Vaccination on HPV Prevalence and Cervical Cancer Incidence in Mali. Clin Infect 52(5): 641-645.

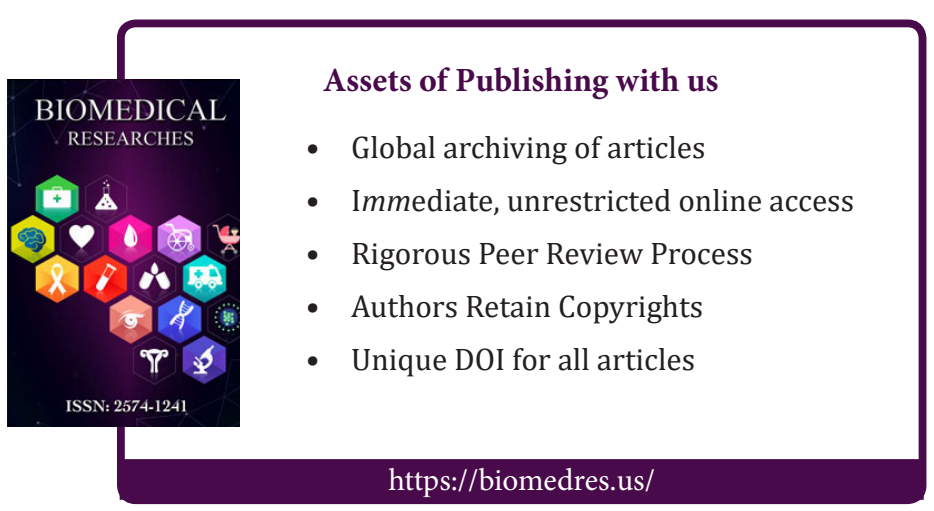

